\title{
Localization and Estimation of Bending and Twisting Loads Using Neural Networks
}

\author{
Diyar Khalis Bilal, Mustafa Unel, Mehmet Yildiz, Bahattin Koc \\ Faculty of Engineering and Natural Sciences, Sabanci University, Istanbul, Turkey \\ Integrated Manufacturing Technologies Research and Application Center, Sabanci University, Istanbul, Turkey \\ \{diyarbilal, munel, meyildiz, bahattinkoc\}@ sabanciuniv.edu
}

\begin{abstract}
In this paper a neural network based modeling approach is proposed for localization and estimation of loads acting on aircraft wings from full field depth measurements. These measurements can be provided by a multitude of sensors such as depth cameras. Depth cameras have many advantages over other intensity sensors in that they can work in low light conditions and they are invariant to texture and color changes. First, an autoencoder is proposed to extract maximum informative data from the depth images and encode them at a much smaller dimension. Next, to develop the models for localization and estimation of loads, supervised multinomial classification and logistic regression networks are proposed, where the encoded depth features are utilized as input in both networks. The performance of the proposed method is validated on a composite wing subject to concentrated and distributed loads, during which the proposed methods for localization and estimation of loads achieved very high accuracies of $94.3 \%$ and $92.7 \%$, respectively.
\end{abstract}

Index Terms-Structural Health Monitoring, Load Localization, Load Estimation, Depth Sensor, Artificial Neural Networks

\section{INTRODUCTION}

One of the major application areas of Structural Health Monitoring (SHM) are aircraft industries due to the operation nature of airplanes. Aircraft wings are structures designed to be the main source of lift generation and they are subject to almost all of the external loads acting on an aircraft. Therefore, the wings are designed to withstand different kinds of external loads through analytical models, but these models are prone to errors due to inaccuracies in the prediction of actual deflections occurring during flight. Therefore, a SHM system for monitoring of loads is vital for these structures to ensure safe operation and to increase their service life.

Current systems for structural health monitoring of aircraft wings utilize strain gauges [1], [2], optical measurement systems [3]-[7] and fiber brag grating (FBG) [8]-[11] sensors.

The SHM systems based on strain gauges are widely used for measuring wing deflections both in literature and in the industry due to their proven high measurement accuracies [1], [2]. However, external temperature variations easily affect them, and they can be easily damaged by physical scratches or cuts. Moreover, if one needs to monitor the whole wing a large number of them must be installed due to their small size.

As for monitoring of loads acting on aircraft wings and measurement of their deflections, many optical SHM systems were proposed in literature. The theoretical foundations of video grammetric model deflection (VMD) measurement was proposed by Burner et al. [3], which was used in wind tunnel testing by National Aeronautics and Space Administration (NASA) [4]. Many works in literature for wing deflection monitoring were motivated by the crash of the unmanned aerial vehicle (UAV) Helios [5], [6]. In the work by Lizotte et al. [7], wing deflection measurements were utilized for estimation of loads acting on aircraft wings. In their work, the deflections were measured through infrared LEDs installed on the wings. However, a many LEDs need to be installed in order to cover the whole wing.

As for the usage of FBGs, Richards et al. [8] proposed a realtime system for in flight wing deflection measurements based on FBGs for Ikhana and Global Observer UAVs. Moreover, Alvarenga et al. [9] also utilized FBGs for realtime wing deflection measurements on lightweight UAVs. Moreover, Ciminello et al. [10] proposed a method for in flight shape monitoring in which a network of FBG sensors were utilized for chord wise strain distribution measurements. Additionally, FBG sensors were utilized in the work by Nicolas et al. [11] for estimating the out of plane loads as well as the shape of the wing deflection. In their work, concentrated and distributed loads were applied on the wings to simulate in flight loading conditions. Their estimated out of plane loads and displacements were withing $4.2 \%$ of the ground truth data measured by strain gauges. Granted that FBGs have advantages over conventional sensors used in SHM systems, external temperature variations easily affect them. Moreover, due to their fragile nature their installation requires special attention [12].

In general, It is observed that a model is used to study the behavior of aircraft wing deflections under different types of loads. However, obtaining physics based models for such systems is hard due to system complexity and uncertainties. This is especially the case when a lot of data is acquired from various sensors, where the inherent sensor noise adds more complexity to the system. In these cases data driven modeling techniques have been found to be more effective since all kinds of sensor errors, sensors noise and uncertainties are already present in the acquired data [13]. One of the most effective data driven modeling techniques has been proven to be artificial neural networks (ANN)s [14]-[17].

In this work, an ANN based approach for localization and estimation of loads acting on aircraft wings from depth 
images is proposed. The proposed methodology is based on the usage of full field depth measurements, therefore a single depth camera capable of full field measurement is enough for monitoring of a single wing. Moreover, depth cameras can be used on all kinds of wings including composites due to optical measurement nature of the sensor. Using the proposed framework, the magnitude of both bending and twisting loads causing wing deflections can be estimated and it is not limited to pure pending as was the case in the work by Nicolas et al. [11]. Moreover, the proposed method is also able to estimate the location of the loads acting on the wing, thus making load localization possible under both bending and twisting loading conditions. Load localization can provide valuable information about the nature of the loads acting on the aircraft wings during flights. Therefore, one can improve the design of the wings based on this new data.

The rest of the paper is structured as follows; In Section II a neural networks based approach for monitoring of loads from depth measurements is proposed. In Section III the experimental setup and the conducted experiment is described. The effectiveness of the proposed approach is validated by an experimental study in Section IV, followed by the conclusion in Section V.

\section{An ANN BASEd Methodology fOR Localization AND ESTIMATION OF LOADS}

This work proposes an ANN based modeling method for localizing the loads acting on aircraft wings and estimating their magnitude from depth images. To develop the model for localization of loads, a supervised multinomial classification network is proposed. As for estimation of the magnitude of loads, a logistic regression network is proposed. Moreover, to extract the most useful features from the depth images, both of the proposed classification and regression networks are preceded with an autoencoder. The proposed method is explained in detail in the following subsections.

\section{A. Data Reduction Using Autoencoders}

In this work, an autencoder [18] network is proposed for reducing the dimensionality of the input depth features and to extract only the most informative data from them. This is because working with depth sensors providing full field measurements is computationally expensive due to the rich but very large sizes of data provided by them. Autoencoders provide an encoded representation of the input at a much smaller dimension while retaining the most critical information. Moreover, to avoid obtaining binary encoded data and obtaining the maximum information from the input features, Kullback-Leibler divergence $\left(K L_{D i v}\right)$ [19] is utilized in this work. Kullback-Leibler divergence enforces the mean and/or standard deviation of the encoded data to be some desired values. Furthermore, logarithmic normalization is proposed to be utilized at the input of the autoencoder to minimize the possible large variance between the values of the input depth measurements. The proposed autoencoder algorithm is given as follows:

$$
\begin{gathered}
Y=\Gamma\left(<\log (X), W_{1}>+B_{1}\right) \\
Z=\Gamma\left(<Y, W_{2}>+B_{2}\right) \\
K L_{D i v}=\alpha_{d} \log \frac{\alpha_{d}}{\alpha}+\left(1-\alpha_{d}\right) \log \frac{1-\alpha_{d}}{1-\alpha} \\
C F_{A E}=\frac{1}{N} \sum_{i=1}^{N}\left(X_{i}-Z_{i}\right)^{2}+\beta K L_{D i v}
\end{gathered}
$$

where $X$ is the input depth vector, $Y$ is the output of the encoder, $Z$ is the output of the decoder, $\Gamma$ is the activation function, $\alpha_{d}$ and $\alpha$ are the desired and actual mean and/or standard deviation of the encoded data, respectively, $W_{1}$ and $W_{2}$ are the weight matrices, $B_{1}$ and $B_{2}$ are the bias vectors, $C F_{A E}$ is the autoencoder cost function to be minimized, and $<\cdot, \cdot\rangle$ is the dot product.

After the dimension of the input depth data is reduced and maximum informative data is obtained using the proposed autoencoder, supervised classification and regression networks can then be utilized for localization and estimation of loads from the encoded data as shown in Figure 1.

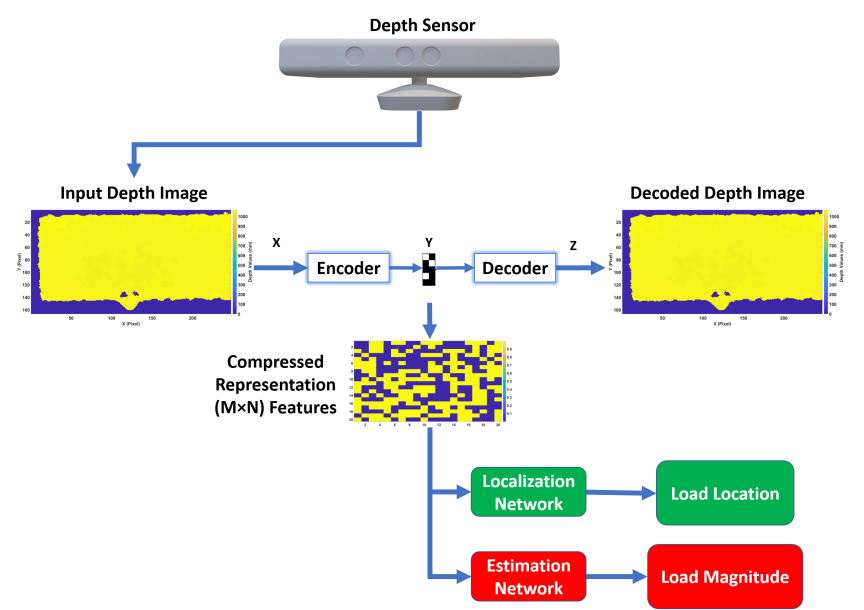

Fig. 1: The proposed framework for localization and estimation of loads using neural networks.

\section{B. Localization of Loads From Depth Images Using Neural Networks}

In this work, a supervised classification ANN is proposed to localize the loads acting on aircraft wings. The input to the proposed localization ANN is provided by the proposed autoencoder in the form of an encoded depth image, which is then used to estimate the location of load. To reduce the effect of sensor noise on the generalization capability of the proposed neural networks, standardization was performed on the encoded depth features for each sample in the training set. This way the input features were made to have zero mean and unit standard deviation. The inputs of the test set were standardized using the mean and standard deviation of the training set. The formula used for standardization is given as follows:

$$
\hat{X}_{i}=\frac{X_{i}-\operatorname{mean}\left(X_{j}\right)}{\sigma\left(X_{j}\right)}
$$


where $X_{i}$ vector contains the encoded features in each sample, $X_{j}$ vector contains the features across the samples, $\hat{X}_{i}$ vector contains the standardized features for each sample and $\sigma$ is the standard deviation.

The standardized input was then fed into the proposed localization network composed of two hidden layers with ReLU (Rectified Linear Unit) activation functions in both layers as shown Figure 2. Two hidden layers with ReLU activation functions were determined to be sufficient for localization of bending and twisting loads from depth images. ReLU was chosen to be used in the proposed classification network instead of other activation functions such as tanh or sigmoid due to its fast convergence.

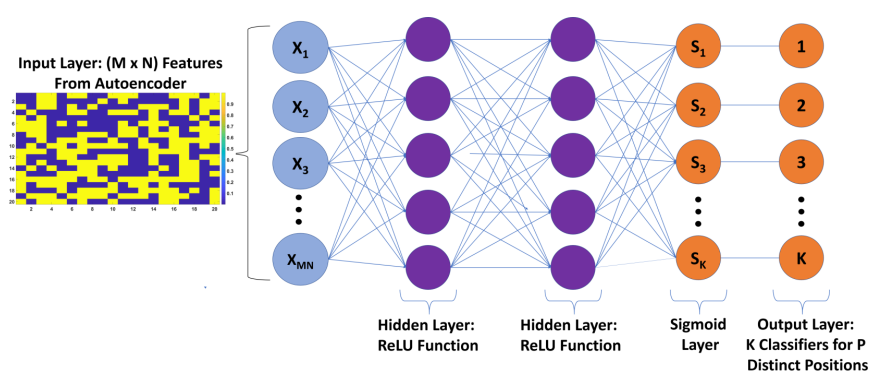

Fig. 2: The proposed load localization ANN with 2 hidden layers and ReLU activation functions.

The output of the second hidden layer was passed through a sigmoid layer to change the arbitrary scores to a range of probabilities ranging between zero and one. Sigmoid instead of other activation functions was used in this layer due to the possibility of loads acting on multiple sections of the wing at the same time. Therefore, the output labels are independent i.e. the output labels are not mutually exclusive and more than one correct label exists in the output. The output labels of the proposed classification network, which are the load positions, were one hot encoded so as to convert categorical data into a numeric one. The closeness between the true labels $(T)$ and the output of the sigmoid layer is defined as cost function. The cost function of the classification $\left(C F_{C L}\right)$ is defined as the average of Cross Entropy Error Function (CEEF) over a batch of multiple samples of size $N$ and labels of size $K$ as follows:

$$
C F_{C L}=\frac{1}{N} \sum_{i=1}^{N} \sum_{j=1}^{K} T_{i j} \log \left(S\left(x_{i j}\right)\right)
$$

Moreover, both dropout and L2 regularization [20] were utilized to prevent overfitting and to increase the generalization capability of the proposed network. This way, the cost function defined by Equation (6) was modified to include a new scalar regularization value $\beta$ due to $\mathrm{L} 2$ regularization. Therefore, the final cost function $F C F_{C L}$ used in the localization ANN is given by Equation (7). The metric used for calculating the accuracy of localization predictions is given by Equation (8). The proposed localization network was trained using Adam [21] optimizer which updates the weights and biases so as to minimize the defined cost function.

$$
F C F_{C L}=C F_{C L}+\beta \sum \| \text { Weights } \|_{2}
$$

$$
\operatorname{Accuracy}_{C L}=\frac{\sum(Y=\hat{Y})}{N} \times 100
$$

where $Y$ is the ground truth, $\hat{Y}$ is the prediction, and $N$ is the number of samples.

\section{Estimation of Loads From Depth Images Using Neural Networks}

For estimation of the magnitude of loads acting on aircraft wings a logistic regression ANN is proposed. The encoded depth images are input to this network and it provides the magnitude of the load as output. The output layer in this network is a single node providing continuous type numeric load and unlike the localization network there is no need for the usage of sigmoid function in the outer layer. The cost function for load estimation $\left(C F_{E}\right)$ is defined as the sum of the squared difference between the ground truth loads and the estimations by the proposed load estimation ANN as given by Equation (9). Similar to the proposed localization network, two hidden layers were determined to be enough for successful estimation of loads. However, the activation functions in this case were chosen as tanh and sigmoid for first and second layers respectively. This network was also trained using Adam [21] optimizer and the accuracy of predictions [22], [23] for load estimation is calculated using Equation (10). The proposed ANN for estimation of loads is shown in Figure 3.

$$
C F_{E}=\frac{1}{N} \sum_{i=1}^{N}(Y-\hat{Y})^{2}
$$

where $Y$ is the ground truth, $\hat{Y}$ is the prediction, and $N$ is the number of samples.

$$
\text { Accuracy }_{E}=\left(1-\frac{\|Y-\hat{Y}\|}{\|Y-\bar{Y}\|}\right) \times 100
$$

where $Y$ is the ground truth, $\hat{Y}$ is the prediction, and $\bar{Y}$ is the mean of the ground truth.

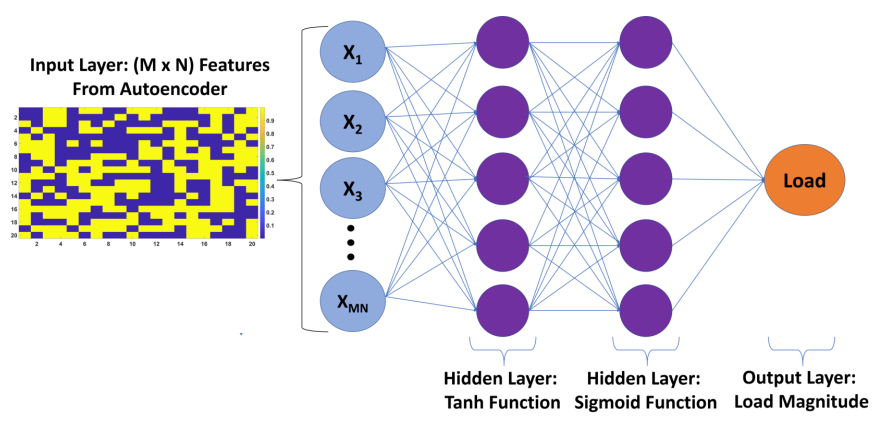

Fig. 3: The proposed ANN for estimation of loads with two hidden layers of tanh and sigmoid activation functions, respectively.

\section{ExPERImental Setup AND Data Collection PROCEDURE}

\section{A. Experimental Setup}

To evaluate the performance of the proposed method for localization and estimation of loads, a quad tilt-wing aircraft [24] made of composites was used in the experiments. The 
dimensions of this aircraft's wing were $50 \times 25 \mathrm{~cm}$ in length and width, respectively. To ensure that no tilting was induced when the loads were applied, the root side of the was fixed. To experimentally mimic the wing deflections that may occur during flights, ground tests were performed similar to the works in the literature. In this experiment both bending and twisting deflections were induced through the usage of calibrated loads in concentrated and distributed loading scenarios. In the concentrated loading case, the loads were made to act on eight different positions of the wing as shown in the left image of Figure 4. As for the distributed loading scenario, the loads were designed to be acting in between the aforementioned eight positions, thus acting on multiple locations of the wing at the same time. This is illustrated in the right image of Figure 4 where thirteen loading positions exist in this case. The magnitudes of the calibrated loads acting on these positions were $[2.45,4.9,7.35,9.81,12.26,14.71] \mathrm{N}$. Therefore, in the concentrated loading scenario six distinct loads were made to act on eight different positions of the wing, which resulted in forty eight loading configurations in this scenario. In the distributed loading case, the same six calibrated loads were made to act on thirteen different positions of the wing, therefore seventy eight loading configurations exist in this loading case. This resulted in application of 126 distinct loading configurations in the conducted experiment.

To measure the wing deflections over its span from a single full field measurement sensor, this work proposes utilization of a depth camera. Depth cameras provide images containing pixel wise depth information of the capture scene. A few of the advantages of depth cameras are that they do not require sophisticated camera calibration procedures, they directly provide $\mathrm{X}, \mathrm{Y}$, and $\mathrm{Z}$ information in the camera frame and unlike conventional cameras they are invariant to color changes and can work in low light conditions [25].
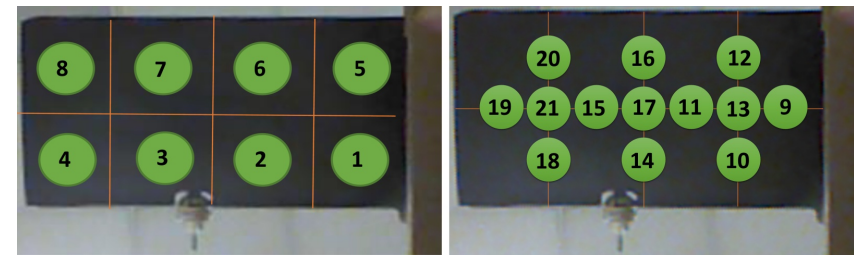

Fig. 4: Positions of concentrated (Left) and distributed (Right) loads (Green) acting on the UAV wing.

The depth sensors to be used in this work was chosen to be Microsoft Kinect V1 [26]. The geometric quality of the depth data collected by Kinect V1 was evaluated by Khoshelham et al. [27]. Based on the results of their analysis, they recommended that the data should be collected at a distance of 1 to $3 \mathrm{~m}$ from the sensor. Therefore, the Kinect V1 sensor in the experiments of this work was placed under the aircraft's wing at a distance of $1 \mathrm{~m}$ as shown in Figure 5. It should be noted that, even though the proposed methodology is evaluated on a relatively small aircraft, the same method can be utilized for monitoring of loads acting on much larger aircraft through the usage of depth cameras with larger field of view such as MYNT EYE [28], Carnegie Robotics ${ }^{\circledR}$ MultiSense ${ }^{\mathrm{TM}}$ S21B [29] and Arcure Omega [30]. The depth camera was not installed on the aircraft in the conducted experiment due to the small size of the used aircraft. Nonetheless, they can be installed on larger aircraft while paying attention to the minimum working distance of the used depth camera. Depth cameras can be installed in place of RGB cameras used in the works by [3], [4], [7] but without the additional requirement of installation of LEDs or markers on the wing.

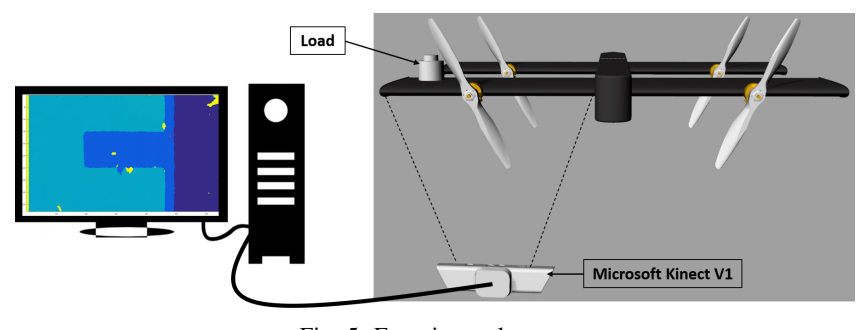

Fig. 5: Experimental setup.

\section{B. Data Collection Procedure}

The data was collected similar to the other works in literature [1]-[3]. After the calibrated loads were applied at each position shown in Figure 4, the depth images of the wing were acquired for a set amount of samples. For instance, the smallest calibrated load was applied on position 1 of the wing only and then the data from the depth image was acquired. This was repeated for all of the other load magnitudes and load locations until data were acquired for all of the load configurations for both concentrated and distributed loading scenarios. The depth images provided by Microsoft Kinect V1 are shown in Figure 6. In this image each pixel value shows the actual measured distance in $\mathrm{mm}$. Due to the distance between the depth sensor and the wing, the acquired images contained depth information of the unnecessary surrounding area of the wing; therefore, the original images with a resolution of $640 \times 480$ pixels were cropped to include only the wing, which resulted in obtaining a depth image of $247 \times 166$ pixels. It is worth noting that the cropping of the image is unnecessary if the wing encompasses the whole depth image. Moreover, to get rid of redundant depth information in the acquired images, any depth values above $2000 \mathrm{~mm}$ were changed to zero as shown in the right image of Figure 6.

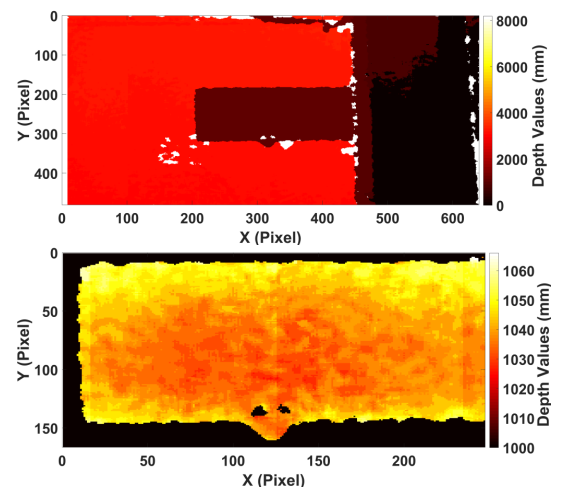

Fig. 6: (Top) Acquired and (Bottom) Cropped depth image of the composite wing. 


\section{DATASET CREATION AND ExPERIMENTAl RESUlts}

\section{A. Dataset Creation}

A dataset of wing deflections due to bending and twisting loads was constructed by following the procedure explained in Section III.B. The Microsoft Kinect V1 was operating @ $30 \mathrm{~Hz}$ during the collection of the data. First, the data was collected when no load was acting on the wing. Then, for concentrated and distributed loading scenarios the depth images were acquired when 6 distinct load magnitudes were being applied at 21 different positions of the wing. For the training dataset, 100 depth images were acquired for each distinct loading case resulting in 12700 samples. The same procedure was performed for the test dataset but this time 30 depth images were acquired for each distinct loading case, which resulted in 3810 samples in this dataset. The constructed training and test datasets are given in Table I. To build and test the proposed ANN models, TensorFlow [31] software was used in this work. The models were developed on a computer having an Intel Xeon 3.6 GHz twelve thread CPU with $16 \mathrm{~GB}$ of RAM without a need for a dedicated GPU.

TABLE I: Training and test datasets

\begin{tabular}{lcccc}
\hline & $\begin{array}{c}\text { Samples per Load } \\
\text { per Position }\end{array}$ & $\begin{array}{c}\text { Distinct Loads } \\
\text { per Position }\end{array}$ & $\begin{array}{c}\text { Distinct } \\
\text { Positions }\end{array}$ & $\begin{array}{c}\text { Total } \\
\text { Samples }\end{array}$ \\
\hline No Load & $100(30)$ & 1 & 1 & $100(30)$ \\
Concentrated Loads & $100(30)$ & 6 & 8 & $4800(1440)$ \\
Distributed Loads & $100(30)$ & 6 & 13 & $7800(2340)$ \\
Training Dataset & 100 & - & 22 & 12700 \\
Test Dataset & 30 & - & 22 & 3810 \\
\hline The ( ) show the number of samples in the test dataset.
\end{tabular}

\section{B. Experimental Results and Discussions}

1) Localization of Loads From Depth Images Using Neural Networks: In this section, the performance of the proposed framework for localization of loads is analyzed and discussed in detail. First, to extract maximum informative data at a much smaller scale from the depth images an autoencoder was proposed in Section II.A. The proposed autoencoder was run in series with the proposed localization network in order to determine the smallest encoded data size required for successful load localization. This way the accuracy of the localization can be used as a measure of performance for the proposed autoencoder. In the localization of concentrated loads, the output positions were labeled 1 to 8 and in distributed loading scenario the labels 9 to 21 were used. The no load condition was labeled as 0 . Thus, 22 distinct positions exist in the output layer of the localization network. These 22 distinct labels were one hot encoded using only nine classifiers in the output of the classification network by choosing $K$ to be 9 and $P$ to be 22. The first eight classifiers were used to one hot encode the labels 1 to 21 , and the ninth classifier was used for the no load case.

The proposed autoencoder and classification ANN was trained using the training dataset described in Section IV.A by varying the size of the encoded data. Initially, the encoded data size was set to have 400 features and then it was incremented by 200 features multiple times until the loads were localized successfully. Sigmoid was chosen as the activation function in the proposed autoencoder. While the first and second hidden layers of the localization network had neurons equal to $60 \%$ and $30 \%$ of the encoded data size, respectively. The mean and standard deviation of the encoded data were set to 0.5 and 0.2 , respectively in Equation (3). The dropout ratio and $\beta$ coefficient for regularization were chosen to be 0.8 and 0.1 , respectively. The learning rate was set to 0.0005 and the number of iterations for training the proposed network was set to 8000 for each encoded data size. The obtained results are given in Table II. These results show that as the size of encoded data increases, the variation between the training and test accuracies decrease. Therefore, one can conclude that as the encoded data size increases more distinctive features are extracted from the depth images. As seen from these results, by using an encoded image with 1200 features only, very high accuracies of $96.4 \%$ and $94.3 \%$ can be obtained for localization of loads when evaluated on the training and test datasets, respectively.

\begin{tabular}{cccccc}
\multicolumn{5}{c}{ TABLE II: Accuracy of the proposed localization ANN. } \\
\hline $\begin{array}{c}\text { Encoded } \\
\text { Data Size }\end{array}$ & $C F_{A E}$ & $\begin{array}{c}\text { Layer 1 } \\
\text { Neurons }\end{array}$ & $\begin{array}{c}\text { Layer 2 } \\
\text { Neurons }\end{array}$ & $\begin{array}{c}\text { Training } \\
\text { Accuracy }_{C L}(\%)\end{array}$ & $\begin{array}{c}\text { Test } \\
\text { Accuracy }_{C L}(\%)\end{array}$ \\
\hline 400 & 31.76 & 240 & 120 & 66.1 & 60.3 \\
600 & 31.58 & 360 & 180 & 93.9 & 88.8 \\
800 & 31.05 & 480 & 240 & 95.1 & 91.3 \\
1000 & 29.98 & 600 & 300 & 96.2 & 93.6 \\
1200 & 29.44 & 720 & 360 & 96.4 & 94.3 \\
\hline
\end{tabular}

The predictions of the proposed localization ANN with 1200 encoded data size when evaluated on the test dataset are plotted against the ground truth in Figure 7 . Here, the no load case is represented by the zero label, the concentrated load positions are labeled 1 to 8 , the distributed load positions are labeled 9 to 21 and the misclassified outputs not belonging to any of the defined positions are labeled as 22. These results show that the proposed localization ANN is capable of classifying the positions of the applied loads causing various bending and twisting deflections on the wing quite accurately. Moreover, the proposed method is invariant to the type of the applied load since it successfully localizes both concentrated and distributed loads with a high accuracy of $94.3 \%$.

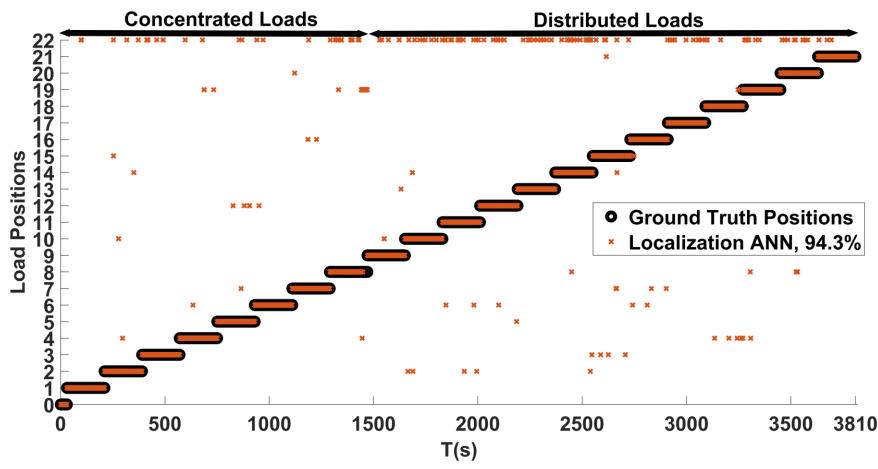

Fig. 7: Position predictions based on the proposed localization ANN evaluated on the test dataset.

2) Estimation of Loads From Depth Images Using Neural Networks: Here, the proposed autoencoder and regression network's load estimation performance is evaluated on the test dataset described in Section IV.A. The output of the proposed regression $\mathrm{ANN}$ is a single continuous variable representing 
the magnitude of both concentrated and distributed loads. The proposed load estimation ANN is preceded with the same autoencoder used in the localization ANN. Moreover, just like the localization of loads, the estimation ANN is trained by varying the size of the encoded data. The training of the estimation network was performed for 30000 iterations for each encoded data size. The obtained results are tabulated in Table III. From these results it is observed that an encoded image with 1200 features again is enough for obtaining very high accuracies of $97.3 \%$ and $92.7 \%$ when evaluated on the training and test datasets, respectively.

\begin{tabular}{cccccc}
\multicolumn{5}{c}{ TABLE III: Accuracy of the proposed load estimation ANN. } \\
\hline $\begin{array}{c}\text { Encoded } \\
\text { Data Size }\end{array}$ & $C F_{A E}$ & $\begin{array}{c}\text { Layer 1 } \\
\text { Neurons }\end{array}$ & $\begin{array}{c}\text { Layer } 2 \\
\text { Neurons }\end{array}$ & $\begin{array}{c}\text { Training } \\
\text { Accuracy }_{E}(\%)\end{array}$ & $\begin{array}{c}\text { Test } \\
\text { Accuracy }_{E}(\%)\end{array}$ \\
\hline 400 & 31.76 & 240 & 120 & 93.9 & 83.1 \\
600 & 31.58 & 360 & 180 & 96.0 & 88.3 \\
800 & 31.05 & 480 & 240 & 96.7 & 90.8 \\
1000 & 29.98 & 600 & 300 & 96.4 & 91.5 \\
1200 & 29.44 & 720 & 360 & 97.3 & 92.7 \\
\hline
\end{tabular}

The predictions by the proposed load estimation ANN with 1200 encoded data size are plotted against the ground truth loads in Figure 8 when evaluated on the test dataset. From these results it is seen that the load estimation ANN is able to estimate the magnitude of both concentrated and distributed loads acting on aircraft wings with a high accuracy of $92.7 \%$.

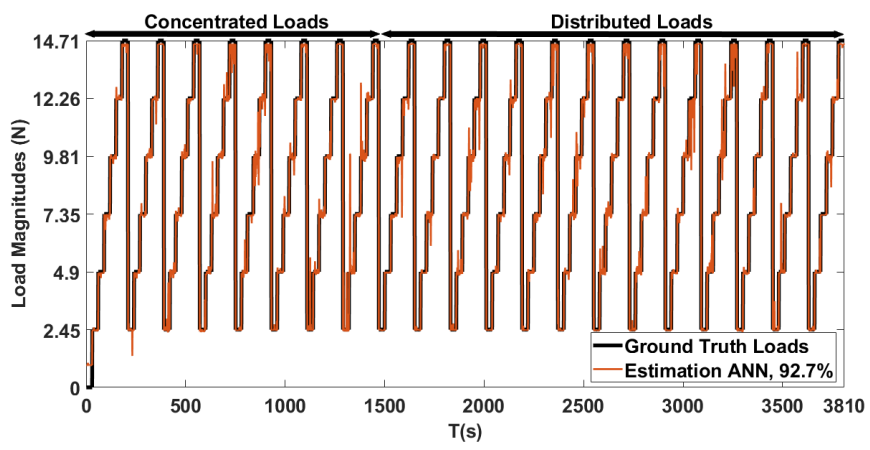

Fig. 8: Load estimation based on the proposed ANN evaluated on test dataset.

As observed from the experimental results, the proposed method can be used for localization and estimation of loads acting on aircraft wings from depth images with very high accuracies. This is valid for loads causing bending and twisting deflections in both concentrated and distributed loading scenarios. It is worth noting that the proposed method was trained with loads causing elastic deflections and it will not work with wings subject to permanent deflections unless it is trained with it as well.

\section{CONCLUSION}

In this work an artificial neural network based load monitoring system for localization and estimation of bending and twisting loads acting on aircraft wings from full field depth measurements is proposed. First, an autoencoder network was proposed to extract the critical information from the full field depth measurements obtained from a depth camera and encode it at a much smaller scale. Then, a supervised classification network composed of two hidden layers with ReLU activation functions was proposed to localize the locations of the loads from the encoded depth images. Moreover, a supervised regression network composed of two hidden layers with tanh and sigmoid activation functions was proposed for estimation of the magnitude of loads from the encoded depth images.

The effectiveness of the proposed framework was validated by an experimental study performed on a composite wing of an UAV, in which both concentrated and distributed loads were applied. The proposed ANN based approach for localization and estimation of bending and twisting loads achieved accuracies of $94.3 \%$ and $92.7 \%$, respectively when evaluated on a dataset containing both concentrated and distributed loads. As shown, the proposed method enables localization and estimation of loads acting on a wing of an aircraft with remarkably high accuracies from a single depth camera.

In the near future, it is planned to extend the current study to localize and estimate dynamic loads with comparison to other state of the art methods.

\section{REFERENCES}

[1] Hong, C. Y., Zhang, Y. F., Zhang, M. X., Leung, L. M. G., Liu, L. Q. Application of FBG sensors for geotechnical health monitoring, a review of sensor design, implementation methods and packaging techniques. Sensors and Actuators A: Physical, 2016.

[2] Ma, Zhen, Xiyuan Chen. Fiber Bragg gratings sensors for aircraft wing shape measurement: Recent applications and technical analysis. Sensors, 2019.

[3] Burner, A. W., Liu, T. Videogrammetric model deformation measurement technique. Journal of Aircraft, 2001,

[4] Burner, A. W., Lokos, W. A., Barrows, D. A. In-flight aeroelastic measurement technique development. In Optical diagnostics for fluids, solids, and combustion II (Vol. 5191, pp. 186-199). International Society for Optics and Photonics, 2003.

[5] https://www.nasa.gov/centers/dryden/news/ResearchUpdate/Helios/

[6] Marqués, P., Da Ronch, A. Advanced UAV Aerodynamics, Flight Stability and Control. Wiley, 2017.

[7] Lizotte, Andrew, William Lokos. Deflection based aircraft structural loads estimation with comparison to flight. 46th AIAA/ASME/ASCE/AHS/ASC Structures. Structural Dynamics and Materials Conference, 2005.

[8] Richards, L., Parker, A. R., Ko, W. L., Piazza, A. Fiber Optic Wing Shape Sensing on NASA's Ikhana UAV. Proceedings of the NAVAIR Meeting. Edwards, CA, USA, 2008.

[9] Alvarenga J., Derkevorkian A., Pena F., Boussalis H., Masri S.F. Fiber optic strain sensor based structural health monitoring of an uninhabitated air vehicle. Proceedings of the International Astronautical Congress. Naples, Italy, 2012.

[10] Ciminello, M., Flauto, D., Mennella, F. FBG sensor system for trailing edge chord-wise hinge rotation measurements. In Sensors and Smart Structures Technologies for Civil, Mechanical, and Aerospace Systems 2013. International Society for Optics and Photonics, 2013.

[11] Nicolas, M. J., Sullivan, R. W., Richards, W. L. Large scale applications using FBG sensors: determination of in-flight loads and shape of a composite aircraft wing. Aerospace, 2016.

[12] Kim, S. W., Kang, W. R., Jeong, M. S., Lee, I., Kwon, I. B. Deflection estimation of a wind turbine blade using FBG sensors embedded in the blade bonding line. Smart Materials and Structures, 2013.

[13] Brotherton, T., Jahns, G., Jacobs, J., Wroblewski, D. Prognosis of faults in gas turbine engines. In 2000 IEEE Aerospace Conference. Proceedings, IEEE, 2000.

[14] Alcan, G. Data driven nonlinear dynamic models for predicting heavyduty diesel engine torque and combustion emissions, $\mathrm{PhD}$ Thesis, Sabanci University, 2019. 
[15] Mumcuoglu, M. E., Alcan, G., Unel, M., Cicek, O., Mutluergil, M., Yilmaz, M., and Koprubasi, K. Driving Behavior Classification Using Long Short Term Memory Networks. In 2019 AEIT International Conference of Electrical and Electronic Technologies for Automotive, IEEE, 2019

[16] Alcan, G., Yilmaz, E., Unel, M., Aran, V., Yilmaz, M., Gurel, C., and Koprubasi, K. Estimating soot emission in diesel engines using gated recurrent unit networks. IFAC-PapersOnLine, 52(5), 544-549, 2019.

[17] Aran, V., and Unel, M. Gaussian process regression feedforward controller for diesel engine airpath. International Journal of Automotive Technology, 19(4), 635-642, 2018.

[18] Hinton, G. E., Salakhutdinov, R. R. Reducing the dimensionality of data with neural networks. science, 2006.

[19] Ng, Andrew. Sparse autoencoder. CS294A Lecture notes 72, 2011.

[20] Srivastava, N., Hinton, G., Krizhevsky, A., Sutskever, I., Salakhutdinov, R. Dropout: a simple way to prevent neural networks from overfitting. The journal of machine learning research, 2014.

[21] Kingma, P. Diederik, Ba. Jimmy. Adam: A method for stochastic optimization. arXiv preprint arXiv:1412.6980, 2014.

[22] Ljung, L. System identification. Springer, 1998.

[23] System Identification Toolbox, Mathworks, 2019.

[24] Cetinsoy, E., Dikyar, S., Hançer, C., Oner, K. T., Sirimoglu, E., Unel, M., Aksit, M. F. Design and construction of a novel quad tilt-wing UAV. Mechatronics, 2012.

[25] Shotton, J., Fitzgibbon, A., Cook, M., Sharp, T., Finocchio, M., Moore, R., Blake, A. Real-time human pose recognition in parts from single depth images, 2011.

[26] https://msdn.microsoft.com/enus/library/jj131033.aspx?f=25

[27] Khoshelham, K., Elberink, S. O. Accuracy and resolution of kinect depth data for indoor mapping applications. Sensors, 2012.

[28] https://www.mynteye.com/products/mynt-eye-stereo-camera

[29] https://arcure.net/omega-stereo-camera-for-indoor-outdoor-applications

[30] https://carnegierobotics.com/multisense-s21b

[31] https://www.tensorflow.org/ 